\title{
Enveloping Subspaces and Superposition of States
}

\author{
Vittorio Cantoni \\ Politecnico di Torino, Torino, Italy
}

\begin{abstract}
In the space of pure states of a generic physical system, a family $\mathscr{L}$ of subsets is singled out and used to extend the quantum-mechanical notion of "superposition" of pure states. $\mathscr{L}$ possesses a natural lattice structure and corresponds to the lattice of closed subspaces of Quantum Mechanics.
\end{abstract}

\section{Introduction}

We have shown in a previous paper [1] that for any physical system described by a set $\mathscr{S}$ of states, a set $\mathcal{O}$ of observables and a probability function $p(A, \alpha, E)$, (the probability that the measurement of the observable $A$ on the state $\alpha$ give a result in the Borel set $E$ of the real line $R$ ), one can define a function $T(\alpha, \beta),(\alpha, \beta \in \mathscr{S})$, which is a generalization of the quantum-mechanical transition probability between pure states.

Let us consider again a physical system with the same degree of generality as assumed in [1], and denote by $\mathscr{S}_{p}$ the set of all its pure states. We shall show that every subset $\mathscr{U}$ of $\mathscr{S}_{p}$ determines a second subset $\tilde{\mathscr{U}}$ of $\mathscr{S}_{p}$ which will be called the enveloping subspace generated by $\mathscr{U}$ and corresponds, in the case of a quantummechanical system, to the closed subspace $U$, in the Hilbert space $H$ of the theory, generated by the representatives of the states belonging to $\mathscr{U}$.

Each subspace $\tilde{\mathscr{U}}$ is associated with a real function $T^{\mathscr{U}}(\alpha, \beta)$ defined in $\mathscr{S}_{p} \times \mathscr{S}_{p}$. In Quantum Mechanics $T^{\mathscr{U}}(\alpha, \alpha)$ is related to the norm of the projection on $U$ of the unit representatives of $\alpha$.

As a consequence it is possible to extend to any physical system the quantummechanical notion of "superposition of pure states", together with an appropriate definition of the "relative phase coefficient" between distinct superpositions of pairs of orthogonal pure states.

It is remarked that the set of all the enveloping subspaces possesses a natural lattice structure, equivalent, in the case of a quantum-mechanical system, to the lattice of closed subspaces of $H$. Such a structure is obtained here with no explicit reference to the lattice of propositions $[3,4,5,6]$. 


\section{Reduced Transition Probabilities and Enveloping Subspaces}

As in [1], our only assumptions on the probability function $p(A, \alpha, E)$ will be Mackey's axioms I and II ([2] p. 62) expressing the fact that, for any fixed observable $A$ and any fixed state $\alpha, p(A, \alpha, E) \equiv \int_{E} d \alpha_{A}$ is a probability measure on the real line, and that the states and the observables are completely characterized by their associated probability measures. Since in the sequel we shall only be concerned with pure states, by "state" we shall always mean "pure state".

Consider an arbitrary subset $\mathscr{U}$ of $\mathscr{S}_{p}$, an observable $A$ and a pair of states $\alpha$ and $\beta$ (not necessarily belonging to $\mathscr{U}$ ). Denote by $E_{A}^{\mathscr{U}}$ the range of $A$ in correspondence with $\mathscr{U}$, defined as the intersection of all the Borel sets $E$ of $R$ such that $p(A, \gamma, E)=1$ whenever $\gamma \in \mathscr{U}$. Set ([1], and [2] p. 100)

$$
T_{A}^{u}(\alpha, \beta)=\left|\int_{E_{A}} d \sqrt{\alpha_{A} \beta_{A}}\right|^{2}
$$

and

$$
T^{\mathscr{U}}(\alpha, \beta)=\inf _{A \in \mathscr{O}} T_{A}^{\mathscr{U}}(\alpha, \beta) .
$$

The function $T^{\mathscr{U}}(\alpha, \beta)$ will be called the reduced transition probability between $\alpha$ and $\beta$ with respect to $\mathscr{U}$.

$T^{\mathscr{U}}(\alpha, \beta)$ is symmetric, not greater than 1 , and $T^{\mathscr{S}_{p}}(\alpha, \beta)$ coincides with the generalized transition probability $T(\alpha, \beta)$ defined in [1]. The relation $T_{A}^{\mathscr{q U}}(\alpha, \beta) \leqq$ $T_{A}^{S_{p}}(\alpha, \beta)$ implies $T^{\mathscr{U}}(\alpha, \beta) \leqq T(\alpha, \beta)$.

In the last relation the equality sign holds whenever one of the arguments, say $\beta$, belongs to $\mathscr{U}$ :

$$
T^{\mathscr{U}}(\alpha, \beta)=T(\alpha, \beta) \quad\left(\alpha \in \mathscr{S}_{p}, \beta \in \mathscr{U}\right) .
$$

Proof.

$$
T_{A}^{\mathscr{S}}=\left|\int_{R} d \sqrt{\alpha_{A} \beta_{A}}\right|^{2}=\left|\int_{E_{A}^{\mathscr{U}}} d \sqrt{\alpha_{A} \beta_{A}}+\int_{R-E_{A}^{\mathcal{U}}} d \sqrt{\alpha_{A} \beta_{A}}\right|^{2} .
$$

If $\beta \in \mathscr{U}$, then $\int_{R-E_{A}^{\vartheta_{A}}} d \beta_{A}=0$, so that $\int_{R-E_{A}^{\mathcal{U}}} d \sqrt{\alpha_{A} \beta_{A}}=0$ : therefore

$$
T_{A}^{\mathscr{S}_{p}}(\alpha, \beta)=\left|\int_{E_{A}^{\mathscr{A}}} d \sqrt{\alpha_{A} \beta_{A}}\right|^{2}=T_{A}^{\mathcal{U}}(\alpha, \beta),
$$

which implies (3).

We shall denote by $\tilde{\mathscr{U}}$ the subset of $\mathscr{S}_{p}$ constituted by the states $\alpha$ such that $T^{\mathscr{U}}(\alpha, \alpha)=1$, and by $\tilde{\mathscr{U}}^{\perp}$ the subset of $\mathscr{S}_{p}$ constituted by the states $\alpha$ such that $T(\alpha, \gamma)=0$ whenever $\gamma \in \mathscr{U}$. $\tilde{\mathscr{U}}$ will be called the enveloping subspace generated by $\mathscr{U}$; $\tilde{U}^{\perp}$ will be called the T-orthogonal complement of $\tilde{\mathscr{U}}$ in $\mathscr{S}_{p}$. We shall also say that two subsets $\mathscr{U}$ and $\mathscr{V}$ of $\mathscr{S}_{p}$ are T-orthogonal if $T(\alpha, \beta)=0$ whenever $\alpha \in \mathscr{U}$ and $\beta \in \mathscr{V}$. In particular two states $\alpha$ and $\beta$ are $T$-orthogonal if $T(\alpha, \beta)=0$.

In the case of a quantum-mechanical system the results of $\lfloor 1\rfloor$ immediately imply that two pure states are T-orthogonal if and only if they are represented by orthogonal rays in the Hilbert space $H$ of the theory.

Let the vector $\underline{\alpha}$ of $H$ be a unit representative of the pure state $\alpha$, and denote by $P_{U}$ the projection operator on the closed subspace $U$ of $H$ generated by the 
representatives of the states belonging to a given subset $\mathscr{U}$ of $\mathscr{S}_{p}$. Then

$$
T^{\mathscr{U}}(\alpha, \alpha)=\left\|P_{U} \underline{\alpha}\right\|^{4} \quad\left(\alpha \in \mathscr{S}_{p}\right),
$$

where $\|\cdot\|$ denotes the norm in $H$.

Proof. Let $\underline{A}$ be the self-adjoint operator associated with a given observable $A$; denote by $\bar{V}$ the minimal closed subspace of $H$ invariant under $A$ and containing $U$, and by $\mathscr{V}$ the subset of $\mathscr{S}_{p}$ represented by $V$. Since the range of $A$ in correspondence with $\mathscr{U}$ is just the spectrum of the restriction of $A$ to $V$, one has $T_{A}^{\mathscr{U}} \equiv T_{A}^{\mathscr{V}}$. On the other hand, if $\underline{\beta}$ is a unit representative of a second state $\beta \in \mathscr{S}_{p}$, the technique used in $[1]$ to express the scalar product $\langle\underline{\alpha}, \underline{\beta}\rangle$ and exhibit the relation $|\langle\underline{\alpha}, \underline{\beta}\rangle|^{2} \leqq$ $T_{A}(\alpha, \beta)$ can now be applied in $V$, practically unchanged, to express $\left\langle P_{V} \underline{\alpha}, P_{V} \underline{\beta}\right\rangle$ and show that $\left|\left\langle P_{V} \underline{\alpha}, P_{V} \underline{\beta}\right\rangle\right|^{2} \leqq T_{A}^{\mathscr{V}}(\alpha, \beta)$, and therefore $\left|\left\langle P_{V} \underline{\alpha}, P_{V} \underline{\beta}\right\rangle\right|^{2} \leqq T_{A}^{\mathcal{U}}(\alpha, \beta)$. In particular $\left|\left\langle P_{V} \underline{\alpha}, P_{V} \underline{\alpha}\right\rangle\right|^{2} \leqq T_{A}^{\mathscr{U}}(\alpha, \alpha)$ and, since $U \subset V$, a fortiori $\left|\left\langle P_{U} \underline{\alpha}, P_{U} \underline{\alpha}\right\rangle\right|^{2} \equiv$ $\left\|P_{U} \underline{\alpha}\right\|^{4} \leqq T_{A}^{\mathscr{U}}(\alpha, \alpha)$. To prove that the last relation holds with the equality sign it is sufficient to choose $\underline{A}$ such that $\underline{A} P_{U} \underline{\alpha}=P_{U} \underline{\alpha}, \underline{A} \underline{\gamma}=0$ whenever $\underline{\gamma} \perp P_{U} \underline{\alpha}$ and $\underline{\gamma} \notin U, \quad \underline{A} \underline{\gamma}=1 / 2 \underline{\gamma} \quad$ whenever $\underline{\gamma} \perp P_{U} \underline{\alpha} \quad$ and $\underline{\gamma} \in U:$ then $p(A, \alpha, \overline{1})=\left\|P_{U} \underline{\alpha}\right\|^{2}$, $p(A, \alpha, 1 / 2)=0$, so that $T_{A}^{\mathscr{U}}(\alpha, \alpha)=\left|\sum_{\lambda=0,1 / 2} p(\bar{A}, \alpha, \lambda)\right|^{2}=\left\|P_{U} \underline{\alpha}\right\|^{4}$ which implies $T^{\mathscr{U}}(\alpha, \alpha) \leqq P_{U} \underline{\alpha} \|^{4}$ and therefore the assertion.

It is now evident that the enveloping subspace generated by $\mathscr{U}$ in $\mathscr{S}_{p}$ corresponds to the closed subspace $U$ of $H$, and that $T$-orthogonal complements in $\mathscr{S}_{p}$ correspond to orthogonal complements in $H$.

\section{Superpositions and Relative Phase Coefficients}

On account of the above considerations it seems natural to regard a state $\alpha$ as a superposition of two states $\mu$ and $v$ if $\alpha$ belongs to the enveloping subspace $\tilde{\mathscr{U}}$ generated by the set $\mathscr{U}=\{\mu, v\}$, and is distinct from $\mu$ and $v$.

In Quantum Mechanics, if $\alpha, \mu$ and $v$ are pure states, the definition just proposed coincides with the usual notion of "superposition". Remaining, for a while, in the context of Quantum Mechanics, assume that $\underline{\mu}$ and $\underline{v}$ are unit representatives of two mutually orthogonal states $\mu$ and $v$ in $H$, and that $\alpha$ and $\beta$ are two distinct superpositions of $\mu$ and $v$ :

$$
\underline{\alpha}=a_{\mu} \underline{\mu}+a_{\nu} \underline{\nu}, \quad \underline{\beta}=b_{\mu} \underline{\mu}+b_{\nu} \underline{\nu} .
$$

In the choice of the unit representatives, the arbitrariness of the phase factors can be exploited in order to make three of the four components, say $a_{\mu}, a_{v}$ and $b_{\mu}$, real and positive: then $b_{v}=\left|b_{v}\right| e^{i \theta}(-\pi<\theta \leqq \pi)$ is well determined. We shall set $\cos \theta \equiv \varrho$, and call $\theta$ the relative phase and $\varrho$ the relative phase coefficient of $\beta$ with respect to $\alpha$ in the $(\mu, v)$-decomposition. One has

$$
\varrho=\frac{|\langle\underline{\alpha}, \underline{\beta}\rangle|^{2}-a_{\mu}^{2} b_{\mu}^{2}-a_{\nu}^{2}\left|b_{v}\right|^{2}}{2 a_{\mu} b_{\mu} a_{v}\left|b_{v}\right|} .
$$

Although the phase factor $e^{i \theta}$ is determined by $\varrho$ only up to complex conjugation, it is possible to give criteria for the correct elimination of this indeterminacy once a convention has been made on the sign of $\theta$ for a particular pair of states $\alpha$ 
and $\beta^{1}$ (the opposite convention would correspond to a physically equivalent representation of the states in a Hilbert space $\bar{H}$ related to $H$ by an antiisomorphism). Thus from the coefficients $\varrho$ and the transition probabilities it is possible to reconstruct, up to unphysical factors, the coordinates of $\underline{\alpha}$ and $\underline{\beta}$ and therefore the physically relevant elements of the Hilbert structure.

We now remark that (5) can also be written in terms of transition probabilities only:

$$
\varrho=\frac{T(\alpha, \beta)-T(\alpha, \mu) T(\beta, \mu)-T(\alpha, \nu) T(\beta, v)}{2[T(\alpha, \mu) T(\beta, \mu) T(\alpha, v) T(\beta, v)]^{1 / 2}} .
$$

In this form the expression for $\varrho$ makes sense for any physical system, whenever $\alpha$ and $\beta$ are superpositions of two T-orthogonal states $\mu$ and $v$.

The characterization of quantum-mechanical systems by this approach is not attempted in this paper.

\section{Remarks on the Lattice Structure of the Family of Enveloping Subspaces}

Let $\mathscr{L}$ be the family of all the subsets of $\mathscr{S}_{p}$ which are enveloping subspaces. $\mathscr{L}$ possesses a natural lattice structure, with the partial ordering defined by inclusion. If $S_{p}$ is regarded as the $T$-orthogonal complement of the void set, the map $\tilde{\mathscr{U}} \rightarrow \tilde{\mathscr{U}}^{\perp}$ is an orthocomplementation of $\mathscr{L}$.

In the case of a quantum-mechanical system the correspondence between T-orthogonality of states and orthogonality of the representative rays implies that $\mathscr{L}$ is isomorphic with the orthocomplemented lattice of closed subspaces of $H$.

Notice that in the present approach the construction of $\mathscr{L}$ involves the transition probabilities only, so that our only assumptions are the very natural axioms I and II of Mackey's on the probability function (Section 2). No explicit reference is made to the lattice of propositions, on which the classical analysis of the structure of Quantum Mechanics are based [3-6].

\section{References}

1. Cantoni, V.: Commun. math. Phys. 44, 125 (1975)

2. Mackey, G.W.: Mathematical foundations of quantum mechanics. New York: Benjamin 1963

3. Birkhof, G., von Neumann, J.: Ann. Math. 37, 823 (1936)

4. Jauch, J.M.: Foundations of quantum mechanics. Reading: Addison-Wesley 1968

5. Varadarajan, V.S.: Geometry of quantum theory, Vol. 1. New York: van Norstrand 1968

6. Piron, C.: Found. Phys. 2, 287 (1972)

Communicated by R. Haag

Received April 5, 1976

\footnotetext{
1 Suppose, for example, that $\underline{\beta}^{\prime}=b_{\mu}^{\prime} \underline{\mu}+b_{v}^{\prime} \underline{y} e^{i \theta^{\prime}}$ ( $b_{\mu}^{\prime}$ positive). Denote by $\varphi$ and $\varrho_{\varphi}$ the relative phase and the relative phase coefficient of $\beta^{\prime}$ with respect to $\beta$, and assume that $\theta$ has been chosen positive, so that $\sin \theta \geqq 0$. Then $\varphi=\theta^{\prime}-\theta, \sin \theta^{\prime} \sin \theta=\varrho_{\varphi}-\varrho \varrho^{\prime}$ and the sign of $\theta^{\prime}$ is determined by the phase coefficients. Analogous criteria can be found if $\alpha, \beta$ and $\beta^{\prime}$ do not belong to the same plane.
} 